\title{
The roles of axes of symmetry in orientation illusions
}

\author{
ALAN A. HARTLEY \\ Scripps College, Claremont, California
}

\begin{abstract}
The lines of a surrounding figure can induce illusory distortion in the apparent orientation of an enclosed line. The axes of symmetry of the figure have been implicated in this distortion. A series of experiments showed generally that the effects are attributable to the axes of bilateral symmetry rather than to less restrictively defined axes of topological symmetry, although those axes may produce other illusory effects. Furthermore, the distortions affect the whole line rather than only parts of it. These results are consistent with interactions of the lines in the orientation rather than position domain. It is suggested that axes of symmetry may play an important role in pattern recognition.
\end{abstract}

When a rod or line enclosed within a frame, a tilted outline figure, is judged to be vertical, it is generally tilted slightly away from the gravitational vertical (Beh \& Wenderoth, 1972; Beh, Wenderoth, \& Purcell, 1971; Witkin \& Asch, 1948). Variations in the magnitude of the error as a function of the shape and tilt of the inducing figure are consistent with the interpretation that settings of vertical are displaced: toward the nearest major frame axis or axis of bilateral symmetry of the figure (Beh \& Wenderoth, 1972; Beh et al., 1971). Wenderoth (1973) has demonstrated that the displacement, termed the major axis effect, can be produced by any axis of symmetry whether it is a physically present line or an implicit, virtual line (see, also, Wenderoth \& Curthoys, 1974).

It has been speculated that the phenomenon may be the result of interactions between the neural representations of the inducing figure and the test line (Wenderoth, 1977; Wenderoth \& Beh, 1977; Wenderoth \& Curthoys, 1974). The interactions may be mediated by a process such as lateral inhibition. The interactions could take place in the orientation domain, as Wenderoth $(1978,1980)$ has argued, in the position domain, or both. The distinction is made between interactions among detectors that respond to the presence of stimuli at specific positions in the visual field and interactions among detectors that respond to the presence of stimuli at specific orientations. Position interactions would likely occur early in the extraction of visual information and might in-

This research was carried out while the author was a visiting faculty member in the School of Social Sciences at the University of California, Irvine. Thanks are due to Joellen Turbitt Hartley and William P. Banks for comments on an earlier version of this report and to Thom Batey for technical assistance. The author's permanent address is Scripps College, Claremont, California 91711. volve a process such as lateral inhibition among retinal receptive fields, which has been offered as a mechanism for aftereffects (e.g., Ganz, 1966) and subjective contours (Coren \& Theodor, 1977). Orientation interactions would occur later and might involve mutual inhibition of orientation-specific cortical cells. It has been proposed that such a mechanism could account for illusory changes in line orientation (Blakemore, Carpenter, \& Georgeson, 1970; Wenderoth, 1977). Interactions in the position domain would require that elements be physically close, while interactions in the orientation domain would require only closely similar orientations. According to the hypothesis of interactions in the orientation domain, the displacements should be the result of distortions in the apparent orientation of the whole test line, and may not require close spatial proximity between inducing figure and test line (Wenderoth, 1978, 1980). According to the hypothesis of interactions in the position domain, the displacements could be the result of distortions in the apparent positions of the points making up portions of the test line. Close proximity between the interacting points would be necessary. Since the interactions are of points, neither the inducing figures nor the variable stimuli need be straight lines.

The major axes of a figure are the axes of bilateral symmetry. It is not implausible that interactions could, in addition, occur in symmetrical portions of a figure that was without an overall axis of bilateral symmetry (see Wenderoth \& Curthoys, 1974, Experiment II). Moreover, interactions in the position domain would not even require local symmetry in a figure. It is possible to define the notion of symmetry less restrictively to allow for these possibilities. A geometry developed by Blum (1973) for the analysis of biological forms provides an alternative conceptualization. Blum's geometry is based on the extraction of symmetric axes from figures. Symmetric axes 
are composed of the sets of centers of the largest disks that will fit inside a figure. The locations of symmetric axes can be determined easily by a physical model. If a grassfire were started along the lines of a figure, then the symmetric axes would be the locations where the fires set along different boundaries would meet and be quenched. For an angle, the quench points lie along the angle bisector; for a circle or arc, the quench point is at the center of curvature. From these examples, it is clear that symmetric axes may be axes of bilateral symmetry but need not be. Errors in judgment produced by orientation interactions should occur only in figures with bilaterally symmetric straight-line components, since only those figures have axes of symmetry. Effects occurring in figures that have symmetric axes-which will be termed axes of topological symmetry-but not axes of bilateral symmetry would be most consistent with interactions in the position domain.

The present experiments, then, seek to determine whether errors in judgment are due to distortions of the orientation of the test line as a whole or of the locations of the points that make up the line, and to examine judgments with inducing figures in which the axes of bilateral and topological symmetry are not identical.

\section{EXPERIMENT 1}

The first experiment was carried out to replicate the earlier findings of an effect on judgment of the major axis of the inducing figure. The inducing figure consisted of two lines that formed a right angle. The bisector of an angle is a major axis; it is an axis both of bilateral and topological symmetry. The figure was oriented so that the bisector, which was not physically present, would be tilted either to the right
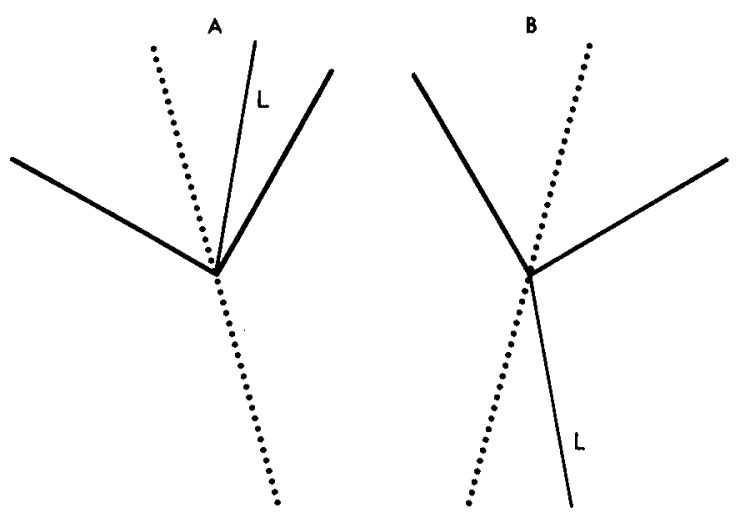

Figure 1. Inducing figures used in Experiment 1. The vertical lines show the subject-adjusted test lines which were either (A) above the vertex of the angle or (B) below it. Axes of bilateral symmetry and of topological symmetry both lie along the dashed lines. The variable lines, $\mathrm{L}$, were to be adjusted to vertical. or to the left of the gravitationally defined vertical (see Figure 1). One end of a variable line was anchored at the intersection of the two lines making up the inducing figure. The line could be rotated, pivoting around the anchored point. If an effect of the major axis were to be observed, then, when judged to be vertical, the variable line would actually be displaced toward the angle bisector. When the variable line lies between the lines of the inducing figure, as in Figure 1a, both the orientation-interaction hypothesis and the position-interaction hypothesis predict such an effect. These conditions were augmented by two in which the variable line fell outside the angle formed by the lines of the inducing figure (as in Figure 1b). An effect in those conditions would be predicted by the orientation-domain hypothesis, but not by the position-domain hypothesis, which would require the inducing figure and the variable line to be in close proximity.

\section{Method}

Apparatus and Stimuli. The stimuli were presented at a distance of $61 \mathrm{~cm}$ on the screen of a Tektronix graphic display terminal (4010) controlled by a Digital Equipment Corporation PDP-11/40 computer. Each subject worked alone in a small, darkened room, although two subjects could be working at one time in different rooms. The intersection of the lines of the inducing figure was centered on the screen, which was $20.8 \mathrm{~cm}$ in width and $16.5 \mathrm{~cm}$ in height $(18.8 \times 15.1 \mathrm{deg}$ of visual angle). The lines of the right-angle inducing figure and the variable line were $6 \mathrm{~cm}(5.6 \mathrm{deg})$ in length and $.5 \mathrm{~mm}(.05 \mathrm{deg})$ in width. The figures were oriented so that the angle bisector was either $15 \mathrm{deg}$ to the left or $15 \mathrm{deg}$ to the right of vertical. To remove cues to verticality provided by the borders of the screen or edges of the terminal, a large, wooden mask was placed over the front of the terminal. The mask was an irregular polygon, about $45 \mathrm{~cm}$ in width at the widest point and $72 \mathrm{~cm}$ in height at the tallest, and was painted flat black. A circular hole, $16 \mathrm{~cm}(14.7 \mathrm{deg})$ in diameter, was cut in the mask. The mask was positioned so that the hole was centered on the display screen.

Procedure. A modified single-staircase procedure was used. On each trial, the subject indicated, by pressing one of two keys, whether the variable line was rotated clockwise or counterclockwise away from vertical. At the outset, the variable line was placed, at random, either 12 deg left or $12 \mathrm{deg}$ right of vertical. Following the subject's response, the variable line was rotated one step in the direction opposite to the judgment. The subject was then asked to judge again. The step size varied, beginning at $10 \mathrm{deg}$ and decreasing by one-half on each response reversal after the third. The procedure was terminated when the step size fell below $.1 \mathrm{deg}$. The instructions explained that the task was to judge whether the variable line was vertical. The concept of vertical was explained with the aid of a plumb line. The subject was instructed that, even if a line appeared to be vertical, a choice must be made between tilted clockwise or tilted counterclockwise from vertical.

The inducing figure could be tilted either to the right or to the left of vertical, and the variable line could be either within or outside the figure for a total of four experimental conditions. Each subject completed two replications of the basic design. The order of conditions was random, with the restriction that one replication be completed before the second was begun. Free inspection of the stimuli was allowed.

Subjects. Fifteen students from introductory social science courses participated in this experiment for extra course credit. 


\section{Results and Discussion}

The analysis was carried out on the discrepancy between the orientation of the variable line at the termination of the staircase procedure and true vertical. In each of the four conditions, the variable line was displaced toward the major axis: When the angle bisector was $15 \mathrm{deg}$ to the left of vertical, the variable line was displayed $1.45 \mathrm{deg}$ counterclockwise from the true vertical toward the bisector when the line was within the inducing figure. It was displaced $1.28 \mathrm{deg}$ counterclockwise toward the extension of the bisector when the variable line was outside the lines of the inducing figure. When the bisector was $15 \mathrm{deg}$ to the right of vertical, a variable line within the figure was displaced 1.58 deg clockwise toward the bisector and a line outside the figure was displaced $1.43 \mathrm{deg}$ toward the extension of the bisector. These figures were reflected in a significant main effect of the tilt of the figure $[F(1,42)=6.44, p<.05]$, with left-tilted figures producing counterclockwise displacements and right-tilted figures producing clockwise displacements. No other significant effects were found.

The displacements observed with the variable line placed between the lines of the inducing figure replicate the earlier findings of a major axis effect. Furthermore, there was an effect of equal magnitude for variable lines that did not fall between the lines of the inducing figure. This result is more readily explained by hypothesizing interactions in the orientation rather than position domains.

\section{EXPERIMENT 2}

The second experiment was carried out to contrast the orientation-interaction and position-interaction hypotheses. The position-interaction hypothesis assumes that the lines of the inducing figure give rise to effects that spread away from their borders. Lateral inhibition, a reasonable candidate for such an effect, has been found to diminish rapidly with increasing distance from the source (see Cornsweet, 1970). In fact, since it is reasonable to assume that the number of interconnections between parts of the retina (and between their cortical representations) decreases as the separation of the areas increases, any such effect would be expected to diminish with distance. This decrease in activation predicts that, with an inducing figure consisting of two lines forming an angle, the interaction in the position domain between the lines of the figure would be strongest close to the intersection of the two lines. The activity would decrease in strength with increasing distance from the intersection, since the lines would be more separated and the effects would be less at the point of summation. Consequently, distortions of the variable line should be greatest close to the intersection of the two lines and should decrease with increasing distance from that point. The orientation-interaction hypothesis

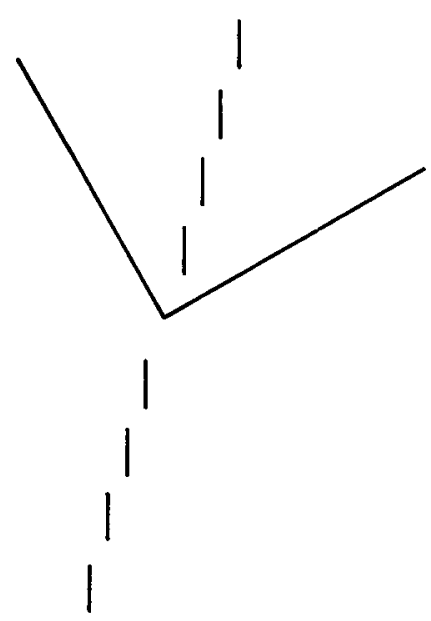

Figure 2. Inducing figure used in Experiment 2, showing the eight locations at which the variable line was placed.

predicts that the distortion in orientation should be the same magnitude everywhere along the axis of symmetry.

\section{Method}

The apparatus and stimuli were the same as in Experiment 1 with the exception of the variable lines. The variable lines were $1.5 \mathrm{~cm}$ (1.4 deg) in length and could be pivoted about their midpoints, which fell along the bisector of the angle, 1.5, 3.0, 4.5, or $6.0 \mathrm{~cm}(1.4,2.8,4.2$, or $5.6 \mathrm{deg})$ from the intersection of the lines of the inducing figure (see Figure 2). These four locations were repeated both within and outside the figure for a total of eight experimental conditions. Since Experiment 1 showed no difference in the magnitude of the effect between inducing figures tilted to the left of vertical and those tilted to the right, only figures tilted to the right were used.

The subject's task, again, was to determine whether the variable line was tilted clockwise or counterclockwise from the gravitational vertical. The staircase procedure used was the same as in the first experiment. Each subject made judgments in each of the eight conditions. The order of conditions was randomly determined. Sixteen persons from the same population as in Experiment 1 participated in the experiment.

\section{Results and Discussion}

The orientation of the line at the end of the staircase procedure was unaffected by the distance from the intersection of the inducing figure's lines $(F<1)$, by whether it was within or outside the figure $[F(1,105)$ $=2.30$, n.s.], or the interaction of those variables $[F(3,105)=2.03$, n.s. $]$. The means for the interaction are given in Table 1 . The overall mean setting was 89.33 deg counterclockwise from the horizontal. This was significantly different from vertical $(90 \mathrm{deg})$ in the direction of the axis of symmetry $[t(15)=3.20$, p $<.01]$.

The results are in general agreement with the prediction of the orientation-interaction hypothesis since the strength of the effect was the same at all the sampled points along the axis of symmetry. The experiment failed to confirm the position-interaction 
Table 1

Mean Vertical Setting (Degrees Counterclockwise from Horizontal): Experiment 2

\begin{tabular}{lcccc}
\hline & \multicolumn{4}{c}{$\begin{array}{c}\text { Distance From Intersection } \\
\text { (in Degrees of Visual Angle) }\end{array}$} \\
\cline { 2 - 5 } $\begin{array}{l}\text { Location of } \\
\text { Test Line }\end{array}$ & 1.4 & 2.8 & 4.2 & 5.6 \\
\hline Between & 89.58 & 89.70 & 89.69 & 88.84 \\
Below & 89.16 & 89.14 & 89.72 & 89.54 \\
\hline
\end{tabular}

Note- "Distance From Intersection" refers to angular separation of the intersection of the lines of the inducing figure and the center of the test line. The test line appeared either between the lines of the inducing figure or below them.

prediction that the effect would diminish with increasing distance from the intersection of the lines of the figure. The magnitude of the effect, an average deviation from vertical of $.66 \mathrm{deg}$, was less than half that found in Experiment 1, in which the average deviation was $1.44 \mathrm{deg}$. This need not be seen as inconsistent with the orientation-interaction hypothesis. Even if physiological mechanisms are not specified, it seems likely that the variability or uncertainty in the extracted orientation of a line would increase as the line became shorter. Greater response from detectors for lines at a variety of orientations, each interacting with responses to the lines of the inducing figures, could reduce the magnitude of the observed effect.

It is important to note that Experiment 2 cannot rule out the possibility that the strength of position interaction does diminish with distance from the intersection of the lines of the inducing figure, but that the strength has reached an asymptote before the first sampled point, $1.4 \mathrm{deg}$ from the intersection. The spread of lateral inhibition from a point of light, for example, is less than a degree of arc in any one direction (Cornsweet, 1970). This interpretation would hold that the errors in setting the entire line to vertical were due to interactions affecting a very small portion of it. (Similarly, Tong \& Weintraub, 1974, argue that the Poggendorf illusion is strongly affected by the small portion of the oblique line proximal to its contact with the parallel.)

\section{EXPERIMENT 3}

The results of Experiments 1 and 2 confirm predictions drawn from the orientation-interaction hypothesis. In both experiments, however, the major axis of the inducing figure was both an axis of bilateral symmetry and an axis of topological symmetry. The clearest tests of the two positions would be in figures having an axis of topological symmetry or an axis of bilateral symmetry, but not both. Experiments 3,4 , and 5 provide such a test. The inducing figure for Experiments 3 and 4 was derived from that of Experiment 1. The straight lines forming the right angle were replaced by arcs (see Figure 3). The figure formed by the intersecting arcs is not bilaterally symmetrical. It does, however, have an axis of topological symmetry along the line equidistant from the two arcs. The position-interaction hypothesis predicts that this axis of symmetry will affect judgments; the orientation-interaction hypothesis makes no prediction.

\section{Method}

The apparatus remained the same as in earlier experiments. The inducing figure consisted of two arcs with chord lengths of $6 \mathrm{~cm}(5.6 \mathrm{deg})$ and radii of curvature of $6 \mathrm{~cm}(5.6 \mathrm{deg})$, meeting at the center of the screen. The chords, which were not visible, formed a right angle with the bisector tilted $15 \mathrm{deg}$ to the right of vertical. The variable line was also an arc that was a segment of a circle whose center of curvature lay on a horizontal line passing through the common intersection of the lines of the inducing figure and the variable line. The subject's task was to decide whether the variable line was bowed to the left of vertical or bowed to the right. Changes in the curvature were accomplished by moving the center of the circle along the imaginary horizontal line, varying its radius. If the intersection of the lines is termed the origin, then the variable line began with the center of the circle randomly placed $60 \mathrm{~cm}(44.5 \mathrm{deg})$ to the left or to the right of the origin. If the variable line was judged to be convex to the left (i.e., the line curved away from vertical to the left), the radius of the circle was increased, moving the center to the left; if judged convex to the right, the center was moved to the right. This reduced convexity in the direction judged. The initial step size for changing the radius of curvature was $12 \mathrm{~cm}(11.1 \mathrm{deg})$. When the radius of curvature exceeded an initial threshold of $90 \mathrm{~cm}(55.9 \mathrm{deg})$, the direction of convexity was changed. Each time the threshold was crossed, the value of the threshold was increased by $50 \%$. On each reversal in direction of judged convexity, the step size was doubled. The procedure was terminated by the subject when he or she judged the variable line to be straight.

Each subject made judgments with the variable line both within the inducing figure and outside the figure. This design was replicated three times, so each subject completed the procedure six times. The order of the conditions was determined randomly.

Eighteen persons from the same population as the earlier experiments participated in the experiment.

\section{Results and Discussion}

The dependent variable for the analysis was the radius of curvature (in $\mathrm{cm}$ ) of the variable line at the conclusion of the staircase procedure. The analysis showed no significant effect of whether the variable
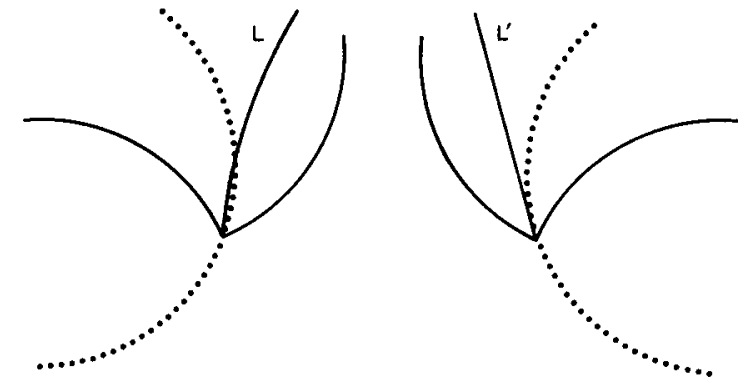

Figure 3. Inducing figure used in Experiment 3. This figure has an axis of topological symmetry, shown by the dashed line, but no axis of bilateral symmetry. Only the figure on the right was used in Experiments 3 and 4. Line $L$ is an example of the variable lines used in Experiment $3 ; L^{\prime}$ is an example of those used in Experiment 4. 
line was between the lines of the figure or outside it $[F(1,85)=1.10$, n.s.], or of replications $[F(2,85)<1]$, or of their interaction $[F(2,85)<1]$. Overall, variable lines within the figure tended to be bowed to the left of vertical, away from the presumed locus of the interactions (radius of curvature, $126.59 \mathrm{~cm}$ ); lines outside the figure tended to be bowed to the right (radius of curvature, $115.85 \mathrm{~cm}$ ). The degree of curvature, the inverse of the absolute value of the mean settings, did not differ significantly from zero (both ts $<1$ ).

The procedures in this experiment represent a significant departure from Experiments 1 and 2 and from previous research on variants of the rod-andframe illusion, all of which have required the subject to set a straight line to vertical. To avoid the possibility that the task may have masked distortions produced by the figure, the experiment was replicated with a straight variable line which the subject was required to set to vertical.

\section{EXPERIMENT 4}

\section{Method}

The methods were identical to those of Experiment 3, with the exception that the subject was to set a straight variable line to be vertical, as in Experiment 1. In addition, two control conditions were added in which no inducing figure was present and the number of replications of each condition was reduced from three to two. Twelve persons from the same population as the previous experiments served as subjects.

\section{Results and Discussion}

The results are in complete agreement with those of Experiment 3. There were no significant effects of the presence or absence of the inducing figure $[F(1,33)$ $=2.27$, n.s.l, of the location of the variable line $[F(1,33)<1]$, or of replications $[F(1,33)<1]$. None of the interactions achieved significance (all Fs $<1$ ). The mean deviation of the final setting was $.17 \mathrm{deg}$ clockwise from vertical; this was not significantly different from zero [t$(11)<1$, n.s.]. Clearly, the judgment required in Experiment 3 was not masking a distortion that would be revealed in judgments of vertical.

\section{EXPERIMENT 5}

Experiment 5 examined judgments in figures with no extended axis of topological symmetry but with a clearly defined axis of bilateral symmetry. Three of the figures are shown in Figure 4; the inducing figure is an arc, and the variable line is straight. The axis of bilateral symmetry of an arc is the line that bisects the chord of the arc and is perpendicular to it. There is actually an axis of topological symmetry, but it is a single point lying at the center of the circle of which the arc would be a part. Because it is a single point, it would be expected to have almost no effect on judgments of

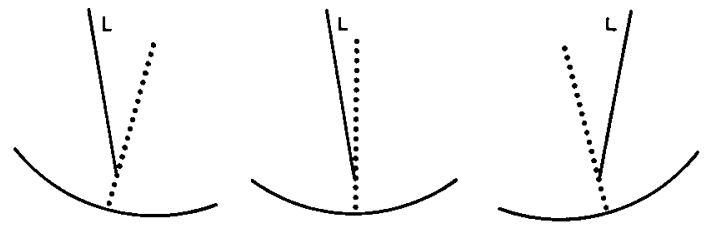

Figure 4. Inducing figures used in Experiment 5. These figures have axes of bilateral symmetry, shown by dashed lines, but no extended axes of topological symmetry. The variable lines, $L$, were to be adjusted to vertical.

much longer lines. More importantly, the radius of curvature of the arcs used was sufficiently large that the axis-point never impinged on the variable line.

\section{Method}

The inducing figures were arcs with radii of curvature of $6 \mathrm{~cm}(5.6 \mathrm{deg})$. The variable line was $4 \mathrm{~cm}(3.8 \mathrm{deg})$ in length and could be rotated about its lower end, which fell at the midpoint of the chord of the arc of the inducing figure. The arcs were oriented so that the axis of symmetry was 7.5, 15.0, or $22.5 \mathrm{deg}$ clockwise or counterclockwise from vertical. Together with one condition in which the axis was vertical, this yielded seven experimental conditions. The conditions were presented in random order to the subject, whose task was to decide whether the variable line was tilted clockwise or counterclockwise from vertical. Nineteen persons from the same population as in the previous experiments served as participants.

\section{Results and Discussion}

A one-way analysis of variance showed that the orientation of the inducing figure significantly affected the settings $[F(6,108)=5.52, p<.01]$. The mean settings, given in Figure 5 , were displaced away from the vertical and toward the axis of symmetry, except when the axis was itself vertical. The displacement increased as the axis was farther from the vertical over the range of tilts studied. When the axis was vertical, the settings were virtually perfect. The results show a clear effect in the absence of any extended axis of topological symmetry.

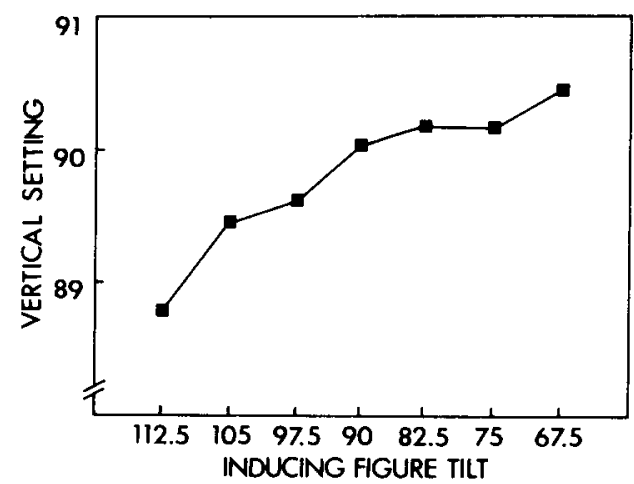

Figure 5. Mean vertical settings as a function of inducing figure tilt in Experiment 5. (Vertical is 90 deg.) 


\section{EXPERIMENT 6}

The procedures used in Experiments 1 to 5 may not have been sensitive enough to detect distortions in small portions of the variable line, even with the small test lines used in Experiment 2. Consequently, Experiments 6, 7, and 8 were carried out, replicating Experiments 1,3 , and 5 , with the difference that subjects were asked to adjust a movable dot until it was vertically above a specified reference point. Wenderoth (1980) has argued that effects of inducing figures on single dots may be independent of their effects on the apparent orientation of whole lines, that interactions in the orientation domain are not simply a summation of interactions in the position domain. If so, we might expect no effects of the inducing lines in these experiments. Alternatively, we might expect results due to position interactions that are unique and different from the results of Experiments 1, 3, and 5 .

In Experiments 6, 7, and 8, the subject was asked to adjust a dot so that it was vertically above a reference point. The first step, then, was to determine whether illusory distortions occur with dots similar to those that occur with lines, whether, when the dot is judged to be vertically aligned with the reference point, it is affected by an axis of bilateral symmetry but not by an axis of topological symmetry.

\section{Method}

The inducing figures in Experiment 6 were right angles, identical to those in Experiment 1. The dots were presented 1.4, $2.8,4.2$, or $5.6 \mathrm{~cm}(1.3,2.6,3.9$, or $5.2 \mathrm{deg})$ above or below the vertex with each of the figure orientations. The four distances above and below the vertex with two orientations resulted in 16 experimental conditions. Distance and location (above or below) were within-subjects variables; orientation of the figure was a between-subjects variable.

The subjects were told that the task was to move the dot horizontally until it was vertically aligned with the vertex of the angle. Again, the concept of vertical was explained with the aid of a plumb line. At the outset of the modified single-staircase procedure, the dot was $1.5 \mathrm{~cm}(1.4 \mathrm{deg})$ to the left or to the right of the true vertical, with the direction determined at random. The subject pressed one of two keys to indicate whether the dot should be moved to the left or the right. The initial step size was $1.2 \mathrm{~cm}$ (1.1 deg). On each reversal in the direction of the response after the second, the step size was halved. By pressing a third key, the subject signaled when the alignment of the dot was satisfactory.

Ten persons from the same population as in the previous experiments served in each of the two orientation conditions for a total of 20 subjects.

\section{Results and Discussion}

The dependent variable was the discrepancy between the actual location of the dot at the end of the staircase procedure and veridical alignment. When a dot was placed to the right of the correct position, the error was designated as positive; otherwise, it was designated as negative. The analyses were actually carried out on the discrepancy measured in units of plotting points on the screen; for clarity, the results are presented here in millimeters.

An analysis of variance showed significant effects of the interaction of inducing figure orientation and location of the dot above or below the figure $[F(1,18)=6.00, p<.05]$. There was also significant interaction among inducing figure orientation, dot location, and distance of the dot from the vertex $[F(3,54)=6.14, p<.01]$. No other effects were significant (all Fs $<1$ ).

Figure 6 shows that, in general, dots were displaced toward the angle bisector. This was true whether they were between the lines of the figure or below the figure. When the bisector was $15 \mathrm{deg}$ counterclockwise from vertical, dots were displaced to the left when located above the vertex and to the right when below; the reverse was true when the bisector was $15 \mathrm{deg}$ clockwise from vertical. The displacement increased as the dot was located farther from the vertex (and therefore farther from the bisector if set to true vertical). The one anomalous data point is that for $5.6 \mathrm{~cm}$ below the vertex with the bisector counterclockwise from vertical. An a posteriori comparison showed that this condition did not differ significantly from the comparable condition, $5.6 \mathrm{~cm}$ above the vertex when the figure was rotated in the opposite direction $[\mathrm{t}(18)=1.40$, n.s.].

The results with dots replicate the distortions found when subjects are asked to set lines to the vertical. When the errors are converted from the linear deviation from a veridical setting to the angular displacement of the implicit dot-to-referencepoint line away from vertical, the mean is $1.91 \mathrm{deg}$. The value is comparable to those obtained in Experiment 1 . The results are compatible with three

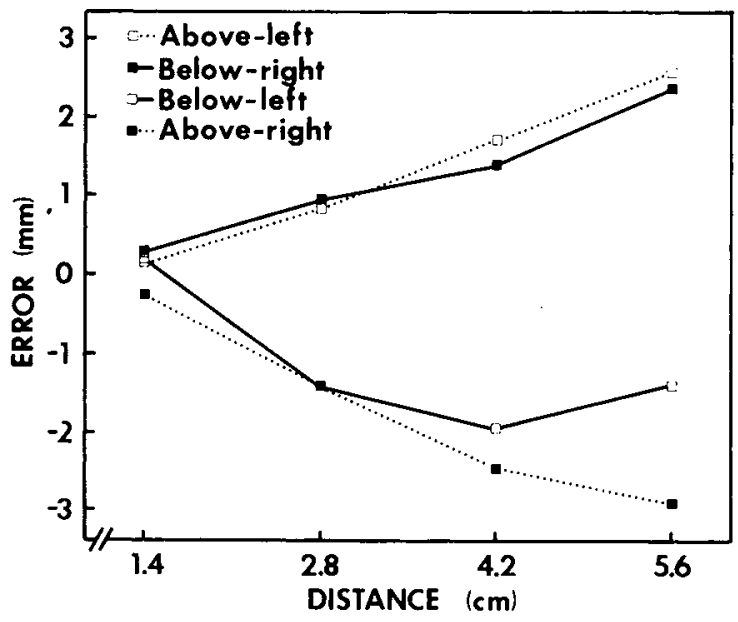

Figure 6. Mean errors (mm) in setting a dot to be vertically above a reference point as a function of inducing figure tilt and dot-to-point distance in Experiment 6. 
conclusions. First, the findings when the variable stimuli were lines in Experiment 1 reflected wholeline distortions, rather than distortions of limited portions of the line. Second, in agreement with the results of Experiment 2, the results are not likely to be due to interactions in the position domain. This is particularly clear for dot positions well away from the intersection of the lines of the inducing figure. The subject attempts to set the implicit line from the dot to the reference point to be vertical. Even though this line is not physically present, the point on it that is present behaves as it would if the entire line were present. Third, single dots do provide adequate probes for illusory distortion, at least with the methods and procedures used here. Wenderoth's (1980) fear that dot placements would not show the effects to which lines are subject is not warranted.

\section{EXPERIMENT 7}

For the inducing figures used in Experiment 6, the axes of bilateral symmetry and of topological symmetry were the same. If the axes of topological symmetry affected judgments, the effects could have been confounded with or overwhelmed by those of the axes of bilateral symmetry. The confounding can be eliminated with the figures used in Experiments 3 and 4, which have axes of topological symmetry but no axes of bilateral symmetry, and those used in Experiment 5, which have axes of bilateral symmetry but no extended axes of topological symmetry. Experiment 7 carried out the first of these tests, using the inducing figures of Experiments 3 and 4 shown in Figure 3.

\section{Method}

With the exception that the inducing figures were intersecting arcs, the method was the same as in Experiment 6 . The figures were oriented so that the bisector of the angle formed by the chords was either $15 \mathrm{deg}$ clockwise or $15 \mathrm{deg}$ counterclockwise from vertical. Sixteen persons from the same population as in the previous experiments served in each of the two orientation conditions, for a total of 32 subjects.

\section{Results and Discussion}

An analysis of variance in the discrepancies showed significant effects of the interaction of direction and distance of the dot from the intersection of the arcs $[F(1,30)=29.16, p<.01]$ and of the interaction of orientation, direction, and distance $[F(3,90)$ $=4.47, \mathrm{p}<.01]$. No other effects were significant. Figure 7 shows the pattern of displacements.

The results did not replicate those of Experiment 3, in contrast to Experiment 6, which produced results analogous to those of Experiment 1 . In Experiments 3 and 4, inducing figures formed of arcs had no effect on the judged straightness or verticality of a variable line. This experiment shows they do affect the judged location of a dot relative

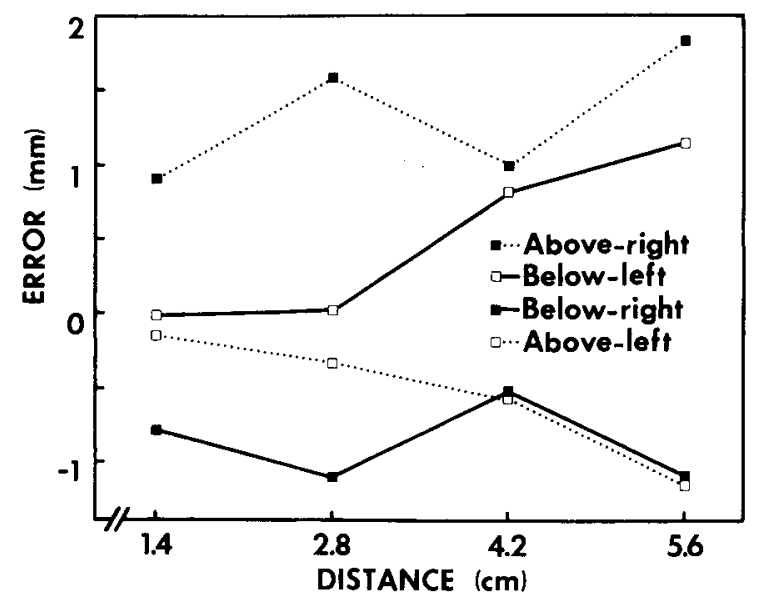

Figure 7. Mean errors $(\mathrm{mm})$ in setting a dot to be vertically above a reference point as a function of inducing figure orientation and dot-to-point distance in Experiment 7.

to a reference point. Moreover, the effect is not limited to regions in which the dot and the lines of the inducing figure are in close proximity. The pattern of displacements is shown in Figure 8. Care should be taken in interpreting the figure, since the displacements have been exaggerated by a factor of 10 relative to the rest of the figure. By speculative analogy with the results of Experiment 6, it is as though the dots were affected by a straight line tilted slightly away from vertical in a direction opposite to the tilt of the figure. A candidate for such a line is the tangent to the axis of topological symmetry where it crosses the intersection of the two arcs of the inducing figure. The tangents are shown by dotted lines in Figure 8 . Suppose that the interaction between the lines occurs only over the small area where they are close, here, an area of less than $1 \mathrm{deg}$ of visual angle in extent. Suppose further that the interaction in this area-which is sufficiently limited that curvature is not detected-triggered line detectors sensitive to that orientation that had rela-
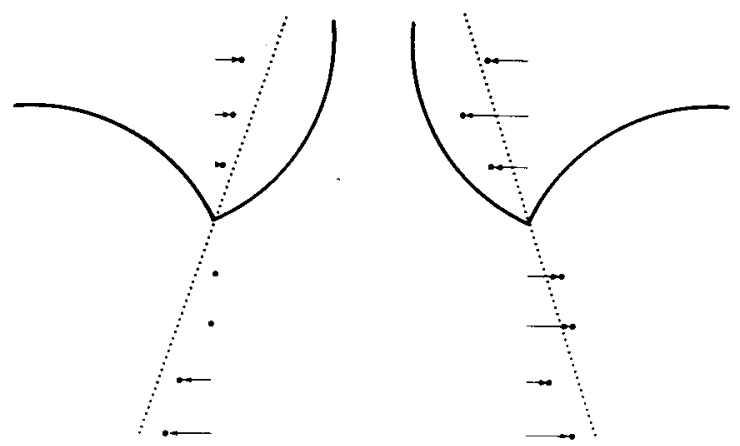

Figure 8. Setting errors (magnified 10 times relative to rest of figure) from Experiment 7, shown superimposed on the inducing figures. Dotted line represents tangent to the axis of topological symmetry, where it intersects the inducing figure. 
tively broad receptive fields. These two suppositions provide an ad hoc, but tenable, explanation for the results. In support of the argument, Walker (1973) showed that postulating activation of neural structures with receptive fields adjacent to those responding to the actual stimulus could account for various illusions of misdirection. In addition, the proposed explanation requires only that interactions occur over a limited distance, consistent with the evidence concerning interactive processes such as lateral inhibition. Nothing in this explanation accounts for the noticeable differences between left- and right-tilted figures.

No distortions were found in Experiments 3 and 4 when the test stimuli were lines; they were found in Experiment 7 with dots. So, while the results of this experiment are consistent with the occurrence of interactions in the position domain, they indicate that these position interactions play, at most, a very small role in distortion of judgments of the orientation of whole lines.

\section{EXPERIMENT 8}

A subset of the figures from Experiment 5 was used to determine whether displacement of single dots occurs when there is an axis of bilateral symmetry but no extended axis of topological symmetry.

\section{Method \\ Three of the arcs used in Experiment 5 were used as inducing figures in this experiment, those with the axis of symmetry $22.5 \mathrm{deg}$ counterclockwise from vertical, vertical, and $22.5 \mathrm{deg}$ clockwise from vertical. The figure was located so that a reference dot, which was placed at the midpoint of the chord, was at the center of the display screen. The dots to be aligned were placed at vertical distances of $1,2,3$, or $4 \mathrm{~cm}(.9,1.9,2.8$, or $3.8 \mathrm{deg})$ above the reference point. Both the orientation of the figure and the distance of the dot from the reference point were within- subjects variables, so each subject aligned dots in 12 conditions. Fifteen persons from the same population as the earlier experi- ments served as subjects.}

\section{Results and Discussion}

Analysis of the variance in the discrepancies showed significant effects of the orientation of the figure $[F(2,154)=30.38, p<.01]$ and of the interaction of orientation and distance of the dot from the reference point $[F(6,154)=5.04, p<.01]$. Figure 9 shows that the dots were displaced toward the axis of bilateral symmetry when the axis was tilted away from the vertical and that the displacement increased as the dot was located farther from the reference point. Despite the effective absence of axes of topological symmetry, there was a clear displacement effect, as found with lines in Experiment 5.

\section{GENERAL DISCUSSION}

The results of the eight experiments provide strong support for the argument that distortions of judgment such as the rod-and-frame illusion are due to

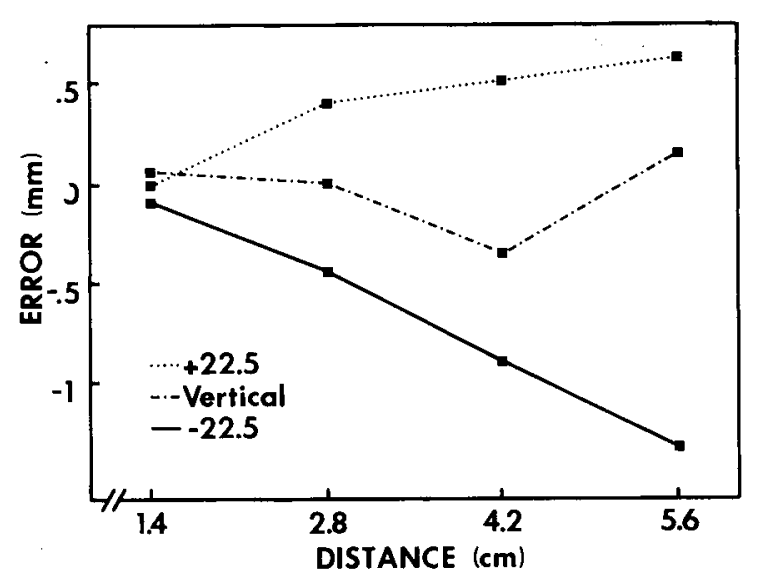

Figure 9. Mean errors ( $\mathrm{mm}$ ) in setting a dot to be vertically above a reference point as a function of inducing figure tilt and dot-to-point distance in Experiment 8.

whole-line effects that occur in the presence of axes of bilateral symmetry. With short lines, the change in orientation was unaffected by the distance from the lines of the inducing figure. With dots as test stimuli, the magnitude of the displacements increased with increasing distance from the axis of bilateral symmetry. Moreover, a similar pattern of displacements with respect to the axis of bilateral symmetry was found for long lines (Experiment 1), short lines (Experiment 2), and dots (Experiment 6), both between the lines of the inducing figure and outside it. The simplest account of the results is that the illusory distortions are produced primarily by interactions between the inducing figure and the test stimulus in the orientation domain. Interactions in the position domain cannot be advanced as a parsimonious account of the results of Experiments 1, 2, 5,6 , and 8 .

Only Experiment 7 produced evidence for distortions of judgment in the absence of an axis of bilateral symmetry. Those effects must be weak, since they did not affect the perceived straightness or curvature of a whole line when the same inducing figures were used in Experiments 3 and 4 . Weak part-line effects may account for the findings that the reproduction of dot locations (Hartley, 1978) and the unconstrained placement of dots within a figure (Psotka, 1978) are affected by axes of topological symmetry. Alternatively, axes of topological symmetry may have qualities that affect memory representations of perceived stimuli or unconstrained judgment but not perceptions of verticality. It should be noted that axes of bilateral and topological symmetry are identical in many figures; in such figures, the axes of topological symmetry would contribute to the distortions generated by the axes of bilateral symmetry.

It is known that the perceived orientation of a line is changed by the proximity of another line at a different orientation (Blakemore et al., 1970; 
Bouma \& Andriessen, 1970; Wenderoth \& White, 1979). It might be argued, then, that the effects that have been observed are due not to the axes of bilateral symmetry directly, but rather to the cumulative change in the perceived orientation of a line induced by two nearby lines at different orientations. There is considerable evidence that this is not the case (Wenderoth, 1973, 1974; Wenderoth \& Beh, 1977; Wenderoth \& Curthoys, 1974; Wenderoth \& White, 1979). For example, Wenderoth (1973) and Wenderoth and Curthoys (1974) showed that a single inducing line tilted $30 \mathrm{deg}$ left or $60 \mathrm{deg}$ right of vertical resulted in an apparent clockwise displacement of a test line. An inducing figure composed of those two lines, however, produced an apparent counterclockwise displacement of the test line.

A second possibility that may be discarded is that the axis of bilateral symmetry has gestalt qualities of good figure. According to this view, the axis attracts the test line or dot that is set to vertical, thus assimilating the figure toward a better gestalt. Such an explanation cannot account for the present results. Experiments 1 and 2 show that the adjustable line is set closer to the axis of symmetry than it would be if truly vertical. This must have been done to compensate for an apparent displacement away from the axis. Thus, the perceptual distortion is in the direction of a poorer, not a better, gestalt. The results are, however, consistent with a response bias distorting settings toward a better gestalt, although it is not clear why a biased setting would be perceived as closer to vertical than would a veridical setting.

A plausible interpretation is that axes of symmetry, extracted by interactions in the orientation domain that operate over broad areas of the retinal field, have perceptual consequences much like physically present facets of the retinal image. It may be that symmetry plays an important role in pattern recognition. Noting that detection of symmetry requires only pairwise comparisons among the simplest features, Julesz, Gilbert, Shepp, \& Frisch (1973) have proposed that discrimination of textual attributes such as symmetry may branch off into a system separate from form recognition at an early level. Symmetry discrimination would be a part of a global texture perception system in contrast to form recognition, which is a local scrutiny system relying on all stages of feature extraction. It is possible to speculate that detection of symmetry is part of a preprocessing (or simultaneous processing) system that facilitates recognition by standardizing the input. Replacing a figure by its axes of symmetry could be used to separate figure and ground, to determine orientation, and to classify input loosely (Blum, 1973; Psotka, 1978).

\section{REFERENCES}

Beh, H., \& Wenderoth, P. M. The effect of frame shape on the angular function of the rod-and-frame illusion. Perception \& Psychophysics, 1972, 11, 35-37.

Beh, H., Wenderoth, P. M., \& Purcell, A. T. The angular function of a rod-and-frame illusion. Perception and Psychophysics, 1971, 9, 353-355.

Blakemore, C., Carpenter, R. H. S., \& Georgeson, M. A. Lateral inhibition between orientation detectors in the human visual system. Nature, 1970, 228, 37-39.

BLum, H. Biological shape and visual science (Part 1). Journal of Theoretical Biology, 1973, 38, 205-287.

Bouma, H., \& ANdriessen, J. J. Induced changes in the perceived orientation of line segments. Vision Research, 1970, 10, 333-339.

Coren, S., \& Theodor, L. H. Neural interactions and subjective contours. Perception, 1977, 6, 107-111.

Cornsweet, T. N. Visual perception. New York: Academic Press, 1970.

Ganz, L. Mechanism of the figural aftereffects. Psychological Review, 1966, 73, 128-150.

Hartley, A. A. The major axis effect: Axes of bilateral symmetry or loci of neural interactions? Perception \& Psychophysics, $1978,23,537-541$.

Julesz, B., Gilbert, E. N., Shepp, L. A., \& Frisch, H. L. Inability of humans to discriminate between visual textures that agree in second-order statistics-revisited. Perception, 1973, 2, 391-405.

Psotka, J. Perceptual processes that may create stick figures and balance. Journal of Experimental Psychology: Human Perception and Performance, 1978, 4, 101-111.

Tong, L., \& We intraub, D. J. Contour displacements and tracking errors: Probing twixt Poggendorf parallels. Perception \& Psychophysics, 1974, 15, 258-268.

Walker, E. H. A mathematical theory of optical illusions and figural aftereffects. Perception \& Psychophysics, 1973, 13, 467-486.

WENDERoth, P. M. The effects of tilted outline frames and intersecting line patterns on judgments of vertical. Perception \& Psychophysics, 1973, 14, 242-248.

Wenderoth, P. M. The distinction between the rod-and-frame illusion and the rod-and-frame test. Perception, 1974, 3, 205-212.

WENDEROTH, P. An analysis of the rod-and-frame illusion and its variants. Studies in perception. Perth: University of Western Australia, 1977.

Wenderoth, P. The major-axis effect: Some comments on Hartley's experiment and analysis. Perception \& Psychophysics, 1978, 24, 572-574.

Wenderoth, P. Dot displacements can be deceptive: A reply to Hartley. Perception \& Psychophysics, 1980, 27, 368-369.

Wenderoth, P. M., \& BEH, H. Component analysis of orientation illusions. Perception, 1977, 5, 57-75.

Wende roth, P. M., \& CURThoYs, I. On the non-additivity of visual tilt illusions. Quarterly Journal of Experimental Psychology, 1974, 26, 549-555.

Wenderoth, P., \& White, D. Angle-matching illusions and perceived line orientation. Perception, 1979, 8, 565-575.

W ITKIN, H. A., \& Asch, S. E. Studies in space orientation: III. Perception of the upright in the absence of a visual field. Journal of Experimental Psychology, 1948, 38, 603-614.

(Manuscript received July 27, 1981; revision accepted for publication January 4, 1982.) 